\title{
Quantum for pressure
}

\author{
Jay Hendricks tells about ongoing work to change the realization and dissemination of the pascal, which \\ will lead to the elimination of mercury-barometer pressure standards.
}

$\mathrm{P}$ ressure is one of the most widely measured quantities in everyday life and is critical to processes from simply taking a breath to weather forecasting or flying an airplane. Yet the way we meausre pressure today is essentially unchanged since the 1640s, when Evangelista Torricelli invented the mercury barometer.

Indeed, primary pressure standards, as curated at the National Institute of Standards and Technology (NIST) and other national metrology institutes (NMIs), still deliver pressure measurements by reading a mercury column's height, along with liquid density and gravity. The instruments are large, heavy and complex, and mercury is neurotoxic. Although digital pressure transducers (which rely on changes in the resistance, capacitance or frequency of mechanically strained diaphragms) have replaced manometers for most applications in science and industry, they must be calibrated by a chain of traceability to a mercury manometer.

This situation will change on 20 May 2019 with the impending redefinition of the International System of Units (SI), when each of the seven base units will be tied to the exact value of a particular fundamental physical constant.

In that context, if a new technique for realizing a unit relies on a quantum property, theoretical calculation or invariant of nature, then that technique could itself serve as a primary standard with direct traceability to the SI. It would not need calibration, and would serve as both a sensor and a standard. This will be the case for the new pressure standard being developed by a team at NIST (Patrick Egan, Jacob Ricker, Jack Stone, Greg Scace, Kevin O. Douglass, Greg Strouse and me).

Pressure is the amount of force per unit area, and hence the pascal $(\mathrm{Pa})$ - the SI unit of pressure - is 1 newton per square meter $\left(\mathrm{N} \mathrm{m}^{-2}\right)$. But pressure can also be expressed as the amount of energy per unit volume $\left(\mathrm{J} \mathrm{m}^{-3}\right)$, because the SI unit of energy, the joule $(\mathrm{J})$, is defined as 1 newton meter.

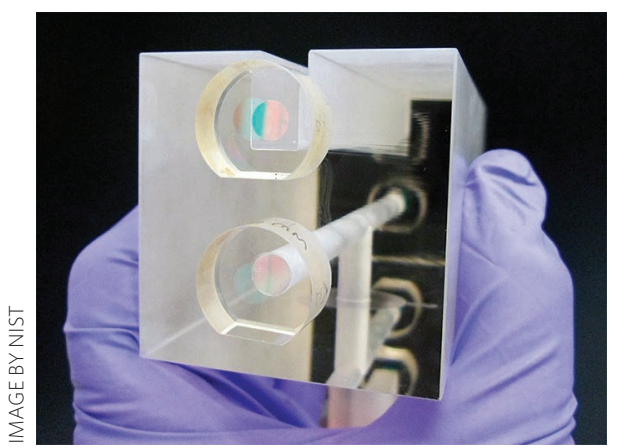

The equivalence of pressure $p$ and energy density provides an alternative measurement method. For an ideal gas, the relation $p=(N / V) k_{\mathrm{B}} T$ holds (the ideal-gas law), where $N$ is the number of particles occupying volume $V, k_{\mathrm{B}}$ is the Boltzmann constant and $T$ is the temperature. The number density $N / V$ of a gas can be obtained via gas refractivity; the equation for pressure then becomes $p \propto(n-1) k_{\mathrm{B}} T$, where $n$ is the refractive index of the gas.

Researchers realized that first-principles calculations of the refractive index of helium could be a new route to realizing the pascal. The NIST team worked with the University of Delaware to determine a high-quality theoretical value based on quantum-chemical calculations ${ }^{1}$. This way, any measurement of the refractive index of helium can be a primary measurement of pressure and the pascal need no longer be defined by a mercury manometer.

The NIST team designed, built and tested a fixed-length optical cavity (FLOC, pictured) for making precise determinations of the refractive index. The FLOC consists of a pair of Fabry-Pérot cavities within a single block of ultralow-expansion glass. The top cavity is filled with gas; the lower is maintained at vacuum. The change in path length between the two cavities depends on the gas's refractive index, density, and atomic or molecular properties. Helium's atomic properties were calculated from first principles, so the refractivity measurement leads to a determination of density, which provides a determination of pressure.

The FLOC further enabled us to determine nitrogen's refractive index with a precision of $1 \times 10^{-6}$ at atmospheric pressure $^{2}$, exceeding the performance of mercury manometers ${ }^{3}$. The NIST team also used helium's theoretical refractive index to make an independent measurement of the Boltzmann constant ${ }^{4}$. The FLOC has smaller uncertainties than a mercury manometer at low pressures and will achieve parts-permillion uncertainties at pressures above the range of manometers.

The NIST team is currently working on a next-generation variable-length optical cavity to enable experimental measurements of nitrogen's refractive index independent of the mercury manometer. By combining this measurement with the theoretically obtained value for helium, the FLOC provides a new way of realizing and disseminating pressure.

The world metrology community is now moving toward the quantum realization of the pascal, and other NMIs are joining the search for primary methods of pressure realization by optical techniques ${ }^{5}$. Continued progress will not only make all quantum-based pressure realizations and measurements directly traceable to the SI, but will finally end the fourcentury-long dominance of mercury in pressure metrology.

JAY HENDRICKS is at the Thermodynamic Metrology Group at the National Institute of Standards and Technology (NIST), Physical Measurement Laboratory, Gaithersburg, Maryland 20899, USA.

e-mail: jay.hendricks@nist.gov

\footnotetext{
References

1. Puchalski, M., Piszczatowski, K., Komasa, J., Jeziorski, B. \& Szalewicz, K. Phys. Rev. A 93, 032515 (2016).

2. Egan, P. F. et al. Opt. Lett. 40, 3945-3948 (2015).

3. Egan, P. F., Stone, J. A., Ricker, J. E. \& Hendricks, J. H. Rev. Sci. Instrum. 87, 053113 (2016).

4. Egan, P. F., Stone, J. A., Ricker, J. E., Hendricks, J. H. \& Strouse, G. F. Opt. Lett. 42, 2944-2947 (2017).

5. Jousten, K. et al. Metrologia 54, S146-S161 (2017).
}

$\mathrm{m} e \mathrm{~A} \mathrm{~s} \mathrm{u} R \mathrm{R} \mathrm{E}_{\mathrm{h}} \mathrm{R} \mathrm{R} \mu_{0} \varepsilon_{0} \alpha \mathrm{R}$

